\title{
Dementia after stroke increases the risk of long-term stroke recurrence
}

\author{
J.T. Moroney, MD, MRCPI; E. Bagiella, MS; T.K. Tatemichi, MD; $\dagger$ M.C. Paik, PhD; Y. Stern, PhD; and \\ D.W. Desmond, PhD
}

\begin{abstract}
Article abstract-Background: Although risk factors for first stroke have been identified, the predictors of long-term stroke recurrence are less well understood. We performed the present study to determine whether dementia diagnosed three months after stroke onset is an independent risk factor for long-term stroke recurrence. Methods: We examined 242 patients (age $=72.0 \pm 8.7$ years) hospitalized with acute ischemic stroke who had survived the first three months without recurrence and followed them to identify predictors of long-term stroke recurrence. We diagnosed dementia three months after stroke using modified DSM-III-R criteria based on neuropsychological and functional assessments. The effects of conventional stroke risk factors and dementia status on survival free of recurrence were estimated using Kaplan-Meier analyses, and the relative risks (RR) of recurrence were calculated using Cox proportional hazards models. Results: Dementia $(\mathrm{RR}=2.71,95 \% \mathrm{CI}=1.36$ to 5.42$)$; cardiac disease $(\mathrm{RR}=2.18, \mathrm{CI}=1.15$ to 4.12$)$; and sex, with women at higher risk $(\mathrm{RR}=2.03, \mathrm{CI}=1.01$ to 4.10$)$, were significant independent predictors of recurrence, while education $(\mathrm{RR}=$ $1.90, \mathrm{CI}=0.77$ to 4.68$)$, admission systolic blood pressure $>160 \mathrm{~mm} \mathrm{Hg}(\mathrm{RR}=1.80, \mathrm{CI}=0.94$ to 3.44$)$ and alcohol intake exceeding 160 grams per week ( $R R=1.86, \mathrm{CI}=0.79$ to 4.38 ) were weakly related. Conclusions: Our results suggest that dementia significantly increases the risk of long-term stroke recurrence, with additional independent contributions by cardiac disease and sex. Cognitive impairment may be a surrogate marker for multiple vascular risk factors and larger infarct volume that may serve to increase the risk of recurrence. Alternatively, less aggressive medical management of stroke patients with cognitive impairment or noncompliance of such patients with medical therapy may be bases for an increased rate of stroke recurrence.
\end{abstract}

NEUROLOGY 1997;48:1317-1325

A number of studies have reported an elevated risk of long-term stroke recurrence following cerebral infarction. The Framingham Study ${ }^{1}$ reported a fiveyear stroke recurrence rate of 42 percent while the Stroke Data Bank ${ }^{2}$ reported a two-year rate of 14 percent. Recent community-based studies have reported five-year recurrence rates ranging from 25 to 30 percent. ${ }^{3,4}$ In contrast to stroke incidence rates, ${ }^{5}$ stroke recurrence rates have not declined, ${ }^{6}$ and with increasing numbers of stroke survivors, there is a significant need to identify the predictors of recurrence. While few risk factors for stroke recurrence have been consistently identified, it is likely some of them will differ from the recognized risk factors for first stroke. Identification of patient groups at increased risk of stroke recurrence may permit targeted application of secondary prevention measures to reduce recurrence rates.

While the prevalence ${ }^{7}$ and incidence ${ }^{8,9}$ of dementia following stroke are high and survival is significantly reduced among stroke patients with dementia, ${ }^{10}$ the prognostic implications of cognitive impairment after stroke with regard to the risk of stroke recurrence have not been investigated. Thus, we performed the present study to determine whether dementia diagnosed three months after ischemic stroke onset would be an independent risk factor for longterm stroke recurrence.

Methods. Subjects. Subjects for this study were recruited among patients consecutively admitted within 30 days of onset of ischemic stroke to Columbia-Presbyterian Medical Center. Eligible patients were 60 years of age or older, spoke English or Spanish as a primary language, and had a diagnosis of acute ischemic stroke of any type. Index ischemic stroke was defined as the acute onset of a focal neurologic deficit attributable to vascular disease of the brain and supported by CT scan (normal or relevant infarct) performed within one week of symptom onset. $\mathrm{Pa}$ tients with mild to moderate degrees of aphasia were included, but those with severe aphasia were excluded if they scored $<2$ on the severity rating scale of the Boston Diagnostic Aphasia Examination (BDAE). ${ }^{11}$ Additional exclusions were the presence of a concomitant primary neurologic disorder potentially affecting cerebral function (e.g., Parkinson's disease), primary language other than English or Spanish, or severe medical comorbidity (e.g., terminal

\footnotetext{
† Deceased April 22, 1995.
}

From the Departments of Neurology (Drs. Moroney, Tatemichi, Stern, and Desmond) and Psychiatry (Dr. Stern), the Division of Biostatistics (E. Bagiella and Dr. Paik), and the Gertrude H. Sergievsky Center (Drs. Tatemichi and Stern), Columbia University, College of Physicians and Surgeons, New York, NY.

Supported in part by National Institutes of Health grants RO1-NS26179 and PO1-AG07232.

Presented in part at the 47th annual meeting of the American Academy of Neurology, Seattle, Washington, May, 1995.

Received May 28, 1996. Accepted in final form August 14, 1996.

Address correspondence and reprint requests to Dr. Desmond, Stroke and Aging Research Project, Neurological Institute, 710 West 168th Street, New York, NY 10032. 
cancer) limiting survival over the planned follow-up period of five years. Informed consent was obtained from subjects or their family members using procedures approved by the Institutional Review Board of Columbia-Presbyterian Medical Center.

During hospitalization, medical and neurologic histories were collected and each patient underwent structured neurologic examinations, cognitive assessment using the MiniMental State Examination (MMSE), ${ }^{12}$ and functional assessments using the Barthel Index ${ }^{13}$ and the Blessed Functional Activity Scale (BFAS). ${ }^{14}$ A more extensive description of our recruitment procedures is available in an earlier publication on methods and baseline findings. ${ }^{7}$ Among the 297 patients who were initially enrolled, 251 received assessments that were adequate for the determination of dementia status three months after stroke, while 9 of those 251 patients were excluded due to early recurrence (i.e., less than 90 days from the index stroke), leaving 242 patients eligible for this study. The mean age of these subjects was $72.0 \pm 8.7$ years and their mean education was $10.1 \pm 4.6$ years. Males comprised $47.1 \%$ of the sample and the cohort was of mixed race-ethnicity with $38.8 \%$ black, $34.7 \%$ white, and $26.5 \%$ Hispanic patients.

Dementia diagnosis. Three months after the onset of stroke, subjects were administered a battery of neuropsychological tests developed for the purpose of diagnosing dementia in a bilingual (English and Spanish), multiethnic, elderly population, ${ }^{15,16}$ and the neurologic and functional examinations were repeated. We chose an interval of three months following stroke onset for neuropsychological testing to allow sufficient time for patients to exhibit an essentially stable course. ${ }^{17,18}$ Based on all available information gathered from the neurologic, neuropsychological, and functional assessments administered at the three month visit, dementia was diagnosed using modified DSMIII-R criteria. ${ }^{19}$ We required the presence of memory impairment as well as deficits in at least two other cognitive domains (i.e., orientation, abstract reasoning, language, and visuospatial function), combined with functional impairment unrelated to physical deficits, as documented with the BFAS. The requirement that patients exhibit deficits in memory and at least two other cognitive domains was intended to ensure that patients were not misclassified on the basis of poor performance on any single measure. To diagnose dementia in aphasic patients who were testable, impairment in nonverbal memory was required. Impairment within each cognitive domain was defined by performance on neuropsychological tests falling below predetermined cutoff scores for each of the subtests developed from pilot data. ${ }^{15}$ The use of cutoff scores was intended to ensure that study criteria for the diagnosis of dementia would be objective and replicable. We have reported norms for this battery in our stroke and control subjects and consider the cutoffs to be conservative. ${ }^{16}$ Agreement on the diagnosis of dementia was excellent, with a Kappa of 0.96 based on independent judgments by two raters in a sample of 63 patients. Based on these operationalized criteria, 62 of the 242 patients $(25.6 \%)$ were found to be demented at the three month examination. The Clinical Dementia Rating $^{20}$ was determined for each patient as a measure of dementia severity.

We used historical information obtained from the BFAS, administered at 7 to 10 days after the index stroke, to reflect the functional status of our patients in the twelve months preceding the stroke and to determine the major clinical subtypes of dementia. When there was evidence of functional deficits predating the index stroke, we diagnosed "mixed" dementia (Alzheimer's disease [AD] and stroke). In the absence of pre-existing functional problems, cerebrovascular disease was inferred to be the central mechanism for the dementia syndrome and a diagnosis of vascular dementia $(\mathrm{VaD})$ was made.

Stroke recurrence. The definition of stroke recurrence was similar to that of the index stroke, but we additionally required that the neurologic deficit be clearly different from that of the index stroke, involve a different anatomic site or vascular territory from that of the index stroke, or be of a stroke subtype different from that of the index stroke. ${ }^{2}$ Based on combined clinical, laboratory, and neuroimaging information, the stroke mechanism for each recurrent event was determined using methods modified from the Stroke Data Bank. ${ }^{21}$ Both ischemic and hemorrhagic strokes were included as recurrent cerebrovascular events. Exact dates for all stroke recurrences were recorded and for those patients who had more than one stroke recurrence we used the date of the first recurrence.

Patient follow-up. Patients were re-examined annually with the clinical assessments described above. Scheduling of annual examinations was based on the date of index stroke onset. We performed continuous surveillance using the methods described above to identify cases of stroke recurrence. To maximize follow-up rates, we made visits to the subject's homes or health care facilities if they were unable or unwilling to visit the medical center, and we did not consider them as having refused any interval examination until at least four attempts had been made to contact and examine them. We also relied upon a daily survey of admissions to the medical center, information from six month interval telephone follow-up calls, and, in cases of death, review of medical records and death certificates to screen for stroke recurrence. Whenever possible, patients were examined and brain imaging was obtained at the time of the recurrence to support the diagnosis of recurrent stroke. Each patient was followed up until death or June 30,1993 , the end of the prospective study period.

Statistical methods. We used survival curves obtained by the Kaplan-Meier product-limit method ${ }^{22}$ to estimate the proportion of patients free of recurrence in the overall sample and then in groups stratified by dementia status and other potential predictors. These included demographic variables (i.e., age, education, sex, and race); conventional vascular risk factors (i.e., hypertension; diabetes; cardiac disease, with myocardial infarction, angina, congestive heart failure, valvular heart disease, and atrial fibrillation combined into one variable; hypercholesterolemia; and cigarette smoking, defined as history of smoking at least one cigarette per day for at least one year); clinical features of the index stroke (stroke syndrome classified as major hemispheral versus other, reflecting the range and severity of neurologic deficits; vascular territory classified as internal carotid versus vertebrobasilar; stroke subtype classified as large artery atherosclerosis, lacunar, cardiogenic embolism, or cryptogenic, based on all available diagnostic information from the index stroke evaluation; ${ }^{21}$ and the Barthel Index score received 7 to 10 days after the index stroke as a measure of functional impairment); blood 
pressure readings on admission; and selected laboratory data (i.e., blood glucose on admission and cholesterol level determined 7 to 10 days after stroke).

We chose to include patients with a history of prior stroke or TIA in our analyses because previous studies have identified both prior stroke and TIA as predictors of recurrence $e^{2,23}$ and we wished to examine their role in our sample. In addition, a history of prior stroke is frequently present in hospitalized stroke patients ${ }^{2,3}$ and we sought a sample that would be representative of elderly stroke patients to increase the generalization of our findings. Finally, silent or asymptomatic cerebral infarction is frequently found on brain imaging even when patients have no history of clinically evident stroke, ${ }^{24,25}$ and exclusion of such patients from studies of stroke recurrence would severely reduce the pool of eligible subjects.

To investigate the putative $\mathrm{J}$ - or $\mathrm{U}$-shaped association between alcohol use and stroke risk, ${ }^{26,27}$ alcohol consumption was assessed as part of a structured questionnaire focusing on general medical risk factors, diet, and lifestyle. The amount and category (beer, spirits, wine) of alcohol typically consumed during one week was converted into grams of alcohol, with one standard drink (a can of beer, a glass of wine, or a single measure of spirits) representing the equivalent of 10 grams of alcohol. ${ }^{26}$ Consumption was classified as $>160$ grams and 60 to 160 grams versus $<60$ grams per week.

The cumulative proportions of patients experiencing a stroke recurrence over the follow-up period were determined in groups stratified by the above variables. Survival time was calculated from the date of the three-month examination and statistical significance was determined by the log-rank test. Reasons for censoring included death unrelated to stroke recurrence, subject drop-out, and reaching the end of the study period. The crude incidence rate (IR) of stroke recurrence, stratified by the presence of dementia, was calculated using life-table methods. To estimate the relative risk of stroke recurrence associated with the presence of dementia while adjusting for demographic variables and other potentially relevant factors, we fitted Cox proportional hazards models. ${ }^{28}$ Variables were selected for entry into the model based on univariate results ( $p \leq$ 0.1 ) or a priori hypotheses based on previously published studies.

Results. Univariate analyses. Among the 242 patients, $208(86 \%)$ completed at least one visit after the baseline examination, $176(72.7 \%)$ completed at least two visits, 111 $(45.9 \%)$ completed at least three visits, and $31(12.8 \%)$ completed at least four visits. Deaths unrelated to stroke recurrence occurred in 67 patients $(27.7 \%)$ and only 5 patients $(2.1 \%)$ were lost to any follow-up. Thus, we were able to obtain information on stroke recurrence in the vast majority of patients with our surveillance methods. Median follow-up for the cohort was 34.5 months, with a maximum follow-up of 59 months. Excluding deaths occurring before each planned follow-up interval, the proportion of patients examined among those at risk of stroke recurrence was $93.8 \%$ at year $1,91.3 \%$ at year $2,96.6 \%$ at year 3 , and $94.6 \%$ at year 4 . During 647 person-years of followup, 45 patients had a recurrent stroke, resulting in a cumulative stroke recurrence rate of $24.0 \%$ and an unadjusted IR of 7.0 per 100 person-years. Cumulative stroke recurrence rates stratified by demographic variables, de- mentia status, vascular risk factors, laboratory data, and clinical features of the index stroke are presented in table 1.

Among the demographic variables, we found no effect for age and race but sex did exert a significant influence on the rate of recurrence, with women at elevated risk. Education was of borderline significance, with less educated patients at elevated risk. Dementia was a significant predictor of stroke recurrence, with almost $50 \%$ of demented patients having a recurrent stroke during the follow-up period of 59 months compared to $20 \%$ of the nondemented group. The unadjusted IR of stroke recurrence in the demented group was 12 per 100 person-years, compared to an IR of 6.0 per 100 person-years in the nondemented group. The cumulative proportion of patients surviving free of recurrence stratified by dementia status is presented graphically in the figure. By the log rank test, survival curves for the two groups were significantly different $\left(\chi^{2}=6.76, p=0.009\right)$.

Among vascular risk factors, there was a trend towards a higher rate of recurrence in patients with cardiac disease. Although a history of smoking was associated with a lower rate of recurrence, further analysis revealed a confounding association between smoking and age, with smokers tending to die at a younger age and thus not at risk of stroke recurrence. There was a trend towards a higher rate of stroke recurrence in patients with an admission systolic blood pressure (SBP) $>160 \mathrm{~mm} \mathrm{Hg}$, but a significant effect was not found for an elevated diastolic blood pressure (DBP). Laboratory data and clinical features of the index stroke, including stroke severity, stroke syndrome, vascular territory, and stroke subtype, did not have a significant effect on the stroke recurrence rate. A history of clinically evident prior stroke or TIA failed to significantly affect the stroke recurrence rate.

Multivariate analyses. The initial Cox model included demographic variables alone, with sex and education showing trends towards significance. Women and patients with less than 12 years of education were at higher risk of stroke recurrence in this model. When baseline dementia status was added to this basic demographic model, we identified a significant independent effect of dementia on the risk of recurrence, with an $\mathrm{RR}$ of 2.08 (95\% CI $=1.09$ to 3.99). Age and race were not significant factors in this model. Sex and education retained their borderline effects, with women and patients with less than 12 years of education having elevated risks of recurrence $(\mathrm{RR}=1.75,95 \%$ $\mathrm{CI}=0.91$ to 3.40 , and $\mathrm{RR}=1.92, \mathrm{CI}=0.78$ to 4.70 respectively).

The final model was developed by adding other variables based on improvement in the log-likelihood ratio, as shown in table 2. The Cox analysis including these variables resulted in an $\mathrm{RR}$ of 2.71 (95\% $\mathrm{CI}=1.36$ to 5.42 ) for dementia as an independent predictor of stroke recurrence. Cardiac disease $(\mathrm{RR}=2.18,95 \% \mathrm{CI}=1.15$ to 4.12$)$ was also a significant predictor of stroke recurrence, as was sex, with women at higher risk $(\mathrm{RR}=2.03,95 \% \mathrm{CI}=1.01$ to 4.10 ). In an effort to determine the specific cardiac diagnoses associated with an increased risk of stroke recurrence, we examined individual cardiac conditions in the Cox proportional hazards analysis, but we found no single cardiac condition was an independent predictor of stroke recurrence, probably due to small sample sizes in those 
Table 1 Cumulative stroke recurrence rates ( \pm standard error) by demographic features, dementia status, vascular risk factors, laboratory data, and clinical features of the index stroke

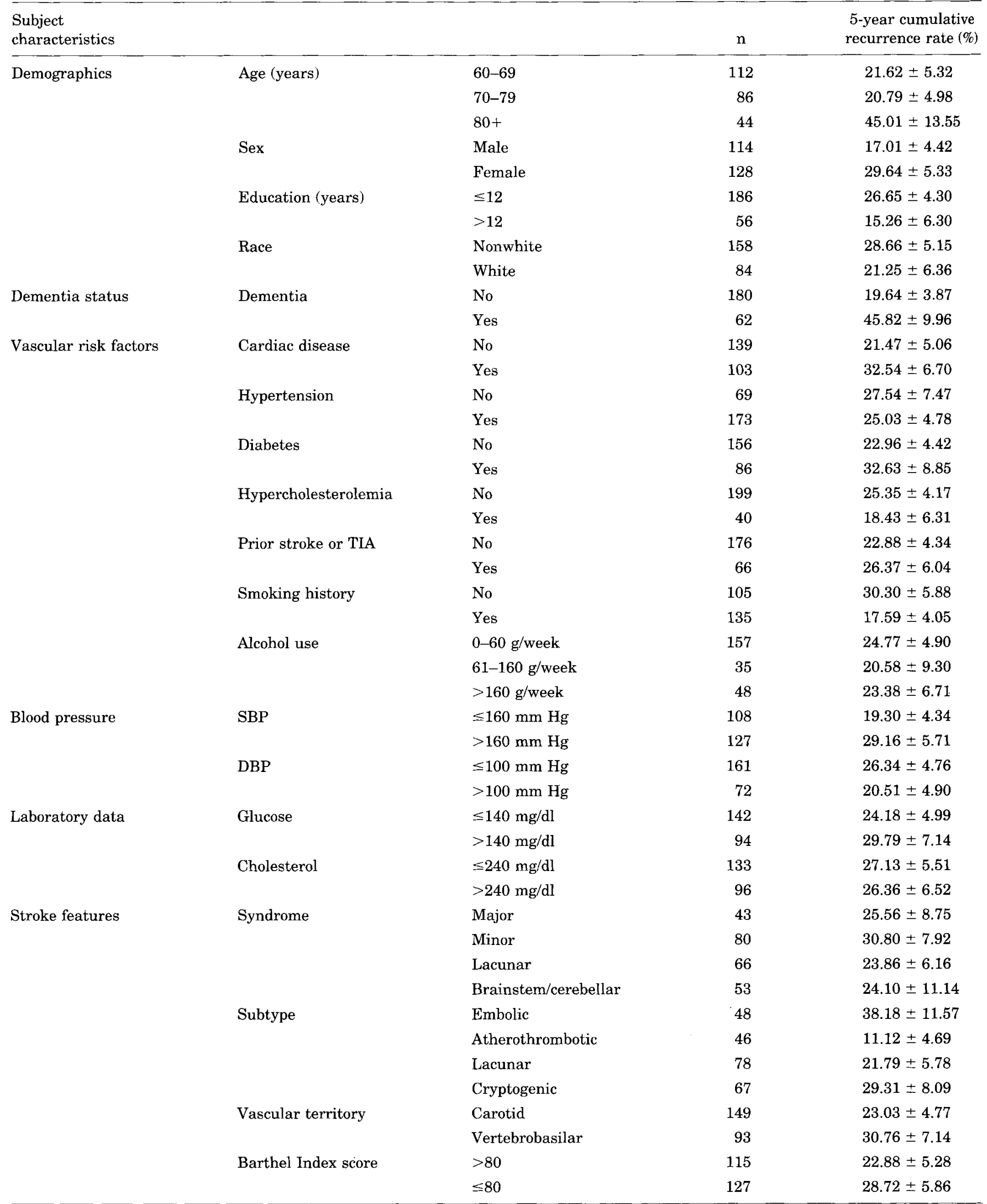

Blood glucose level was measured on admission while cholesterol level was measured 7 to 10 days after index stroke. Some recurrence rates are based on an incomplete sample due to missing data.

$\mathrm{TIA}=$ transient ischemic attack; $\mathrm{SBP}=$ systolic blood pressure; $\mathrm{DBP}=$ diastolic blood pressure. 


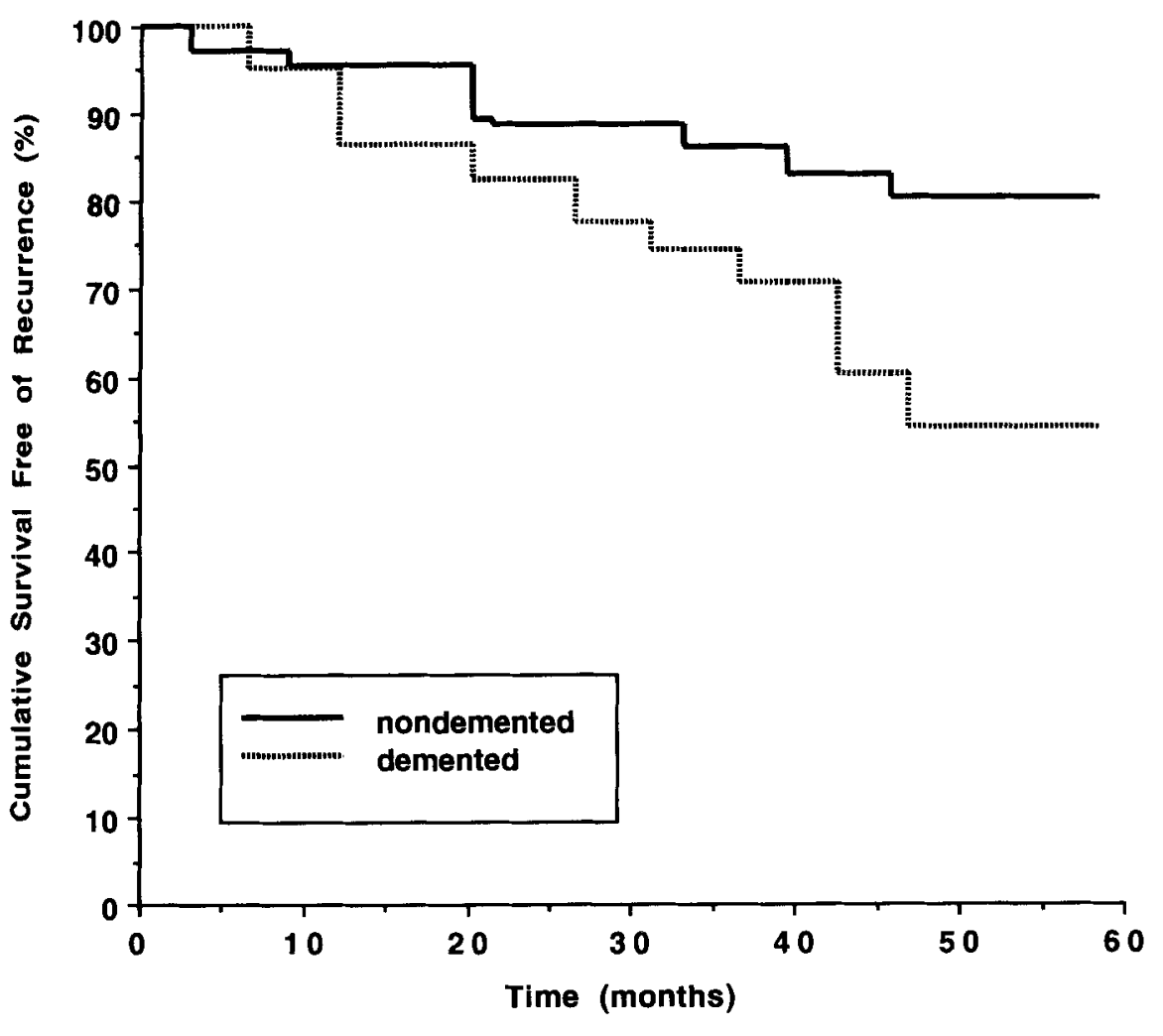

Figure. Kaplan-Meier curves representing the cumulative proportion of patients surviving free of recurrence stratified by dementia status during the follow-up period of up to 59 months. Survival curves are significantly different by the log rank test $(\mathrm{p}=0.009)$. cardiac subgroups. Admission SBP was of borderline significance $(\mathrm{RR}=1.80,95 \% \mathrm{CI}=0.94$ to 3.44$)$ in the final model, as was education, with a higher risk of recurrence in patients with less than 12 years of education $(\mathrm{RR}=$ 1.90 , CI $=0.77$ to 4.68 ). We included alcohol consumption in the multivariate analysis after univariate testing revealed an association between gender and alcohol use and its inclusion improved the log-likelihood ratio of the model.

Table 2 Final Cox proportional hazard model of the relative risk of stroke recurrence for demented stroke patients compared to nondemented stroke patients adjusting for demographic factors and other relevant variables

Relative risk (95\% CI)

Independent variables

Dementia status (yes vs no)

$2.71(1.36-5.42)$

Cardiac disease (yes vs no)

$2.18(1.15-4.12)$

$\mathrm{SBP}$ (>160 $\mathrm{mm} \mathrm{Hg}$ vs $\leq 160 \mathrm{~mm} \mathrm{Hg}$ )

$1.80(0.94-3.44)$

Alcohol consumption $(\leq 60 \mathrm{~g} / \mathrm{wk})$

$61-160 \mathrm{~g} / \mathrm{wk}$

$0.90(0.32-2.49)$

$>160 \mathrm{~g} / \mathrm{wk}$

$1.86(0.79-4.38)$

Covariates

Age (vs 60-69 years)

70-79 years

$1.03(0.49-2.13)$

$80+$ years

$1.06(0.42-2.66)$

Education ( $\leq 12$ years vs $>12$ years)

$1.90(0.77-4.68)$

Sex (female vs male)

$2.03(1.01-4.10)$

Race (white vs nonwhite)

$0.89(0.43-1.85)$

$\mathrm{SBP}=$ systolic blood pressure measured in millimeters of mercury.

Light to moderate drinking (60 to 160 grams per week) was not inversely associated with stroke recurrence $(\mathrm{RR}=$ $0.90,95 \% \mathrm{CI}=0.32$ to 2.49$)$, while heavy drinking $(>160$ grams per week) showed a trend towards significance for an elevated risk $(\mathrm{RR}=1.86,95 \% \mathrm{CI}=0.79$ to 4.38$)$.

Given that we found dementia to be a significant independent predictor of stroke recurrence, we also examined the relationship between the severity of dementia and the magnitude of risk of stroke recurrence. In a Cox analysis, we found that moderate to severe dementia (corresponding to CDR scores of $\geq 2$ ) was a significant independent predictor of stroke recurrence $(\mathrm{RR}=3.03,95 \% \mathrm{CI}=1.33$ to 6.91), while mild dementia (corresponding to a CDR score of 1 ) was weakly related $(\mathrm{RR}=1.58,95 \% \mathrm{CI}=0.70$ to 3.57) after adjusting for demographic variables. Additionally, prior stroke, when considered separately from TIA, was not a significant variable either in our univariate or multivariate analyses and did not significantly alter the calculated relative risks of the predictors when forced into our final multivariate model.

Discussion. Previous studies of outcome after cerebral infarction have tended to focus on survival after stroke, rather than stroke recurrence, ${ }^{29-31}$ while those which have investigated predictors of recurrence have tended to limit their analysis to conventional vascular risk factors. ${ }^{1-4,6}$ Although previous studies have examined the effects of stroke-related cognitive impairment on functional recovery and survival and suggested that stroke survivors with cognitive deficits have a poorer prognosis with regard to those outcomes, ${ }^{10,32-35}$ our study is the first, to our knowledge, to examine the role of cognitive impairment after stroke as a predictor of long-term recur- 
rence. Recurrent stroke was frequent in our sample, with an overall five-year cumulative stroke recurrence rate of $24.0 \%$, which is comparable to that of other studies. ${ }^{3,4}$ We found in our prospective investigation that the incidence of stroke recurrence was approximately doubled among patients with dementia following stroke relative to nondemented patients. Dementia after stroke remained a significant independent risk factor for long-term stroke recurrence after adjusting for demographic variables and other vascular risk factors. We found no effect for race or age on the risk of stroke recurrence, but women and patients with fewer years of education were at higher risk of recurrent stroke in our sample. With regard to vascular risk factors, only cardiac disease was an independent predictor of long-term stroke recurrence, although a trend towards increasing risk was noted for a systolic blood pressure (SBP) $>160 \mathrm{~mm} \mathrm{Hg}$. In addition, we noted a weak trend towards an elevated risk of stroke recurrence for heavy alcohol consumption but no significant protective effect for light to moderate alcohol consumption.

Our finding that dementia is an independent predictor of long-term stroke recurrence is intriguing and could be related to a number of pathogenic mechanisms. First, since stroke itself increases the risk of dementia, ${ }^{7-9}$ and our findings suggest that dementia may also cause stroke, a bidirectional causal link between dementia and stroke may exist, with dementia serving as a surrogate marker for larger infarct volume or multiple vascular risk factors, and, thus, an increased stroke risk. Indirect support for this hypothesis is derived from several sources, including the observations that larger infarct volumes may be associated with dementia after ischemic stroke ${ }^{36,37}$ and that the risk of dementia in stroke populations may be increased by exposure to vascular risk factors such as prior symptomatic stroke, myocardial infarction, and diabetes mellitus. $^{38,39}$ Data from a recently conducted, populationbased, prospective cohort study provides further evidence in support of a bidirectional causal link between cognitive impairment and cerebrovascular disease in the elderly, having found that a low score on a mental status questionnaire was an independent risk factor for stroke incidence in initially stroke-free subjects aged 71 years and older. ${ }^{40}$ Interestingly, the association between cognitive impairment and the risk of a future stroke was independent of the significant effects of hypertension and diabetes. The authors proposed that silent or subclinical cerebrovascular disease may be an underrecognized but important risk factor for cognitive impairment in the elderly and may serve to increase the risk of clinically evident stroke.

Second, the reported association between strokerelated dementia and the presence on CT of prior asymptomatic or silent brain infarcts offers a potential mechanism underlying higher rates of recurrence in demented stroke patients. Among 163 patients with silent brain infarction in the Stroke Data
Bank, dementia was more frequent with increasing number and size of silent lesions, ${ }^{41}$ while in the Stroke and Aging Research Project, we have found multiple lacunar infarcts, both symptomatic and asymptomatic, to be a significant correlate of dementia following ischemic stroke. ${ }^{42}$ The European Atrial Fibrillation Trial (EAFT) reported that patients with silent infarcts on brain CT had a higher risk for both recurrent vascular events in general (vascular death, myocardial infarction, systemic embolism, and stroke) and for recurrent stroke in particular. ${ }^{43}$ Other studies have found the extent of cerebral white matter disease or leukoaraiosis on CT in stroke patients to be an independent risk factor for recurrent stroke and associated with an increased frequency of dementia. ${ }^{44,45}$ Silent brain infarcts and leukoaraiosis may increase susceptibility to strokerelated cognitive decline by reducing cognitive reserve, may be significantly more prevalent in patients with dementia following stroke, and may serve as a marker for a greater burden of cerebral arteriosclerosis, thereby placing these patients at higher risk of recurrent stroke.

Third, dementia may be a predictor of long-term stroke recurrence due to less aggressive medical management of patients with stroke-related cognitive deficits. Diminished intellectual competence following stroke may result in patients being considered poor candidates for secondary prevention programs. Physicians may be reluctant to initiate oral anticoagulation with warfarin, for example, due to the elevated risk of hemorrhage that may result from inadequate monitoring and inconsistent administration in stroke patients with significant cognitive deficits. Alternatively, noncompliance of demented stroke patients with prescribed secondary preventive measures due to cognitive impairment may be a basis for an increased rate of stroke recurrence. In addition, patients with cognitive impairment following stroke are significantly more likely to have functional deficits resulting in nursing home placement or dependent living at home, ${ }^{16}$ potentially compromising medical follow-up and management of hypertension, diabetes, and other chronic vascular risk factors.

We also found cardiac disease to be a significant independent risk factor for long-term stroke recurrence. To represent cardiac disease, we used a variable combining a broad range of cardiac conditions that could cause cardiogenic embolic cerebral infarction. ${ }^{46,47}$ Small sample sizes in our subgroup analyses probably hindered our ability to detect effects for individual cardiac diseases. Marquardsen ${ }^{32}$ found in a retrospective study that congestive heart failure, atrial fibrillation, and electrocardiographic abnormalities were associated with higher rates of recurrence, while in the Stroke Data Bank an abnormal first electrocardiogram was a significant risk factor for stroke recurrence at one year. ${ }^{48}$ The Framingham Study ${ }^{1}$ reported that cardiac conditions such as coronary heart disease and congestive heart failure con- 
tributed significantly to higher five-year cumulative recurrence rates, while investigators in the Lehigh Valley Stroke Study ${ }^{23}$ found an approximately eightfold increased risk of recurrent stroke among patients with myocardial infarction and a pooled grouping of other cardiac diseases. The recent availability of transesophageal echocardiography has added new data in support of an increased risk of recurrent stroke with potential cardiac sources of embolism, such as patent foramen ovale with inter-atrial shunt, left atrial thrombus, and atrial septal aneurysm, and suggests that further studies are needed to determine the optimal management of stroke patients with potential cardiac sources of embolism. ${ }^{49,50}$

The role of hypertension in relation to stroke recurrence is unclear with previous studies reporting conflicting results. ${ }^{1-4,6} \mathrm{We}$ found no effect for hypertension diagnosed prior to the index stroke nor for an elevated DBP on admission, but we noted that an admission SBP $>160 \mathrm{~mm} \mathrm{Hg}$ increased the risk of long-term stroke recurrence. Irie et $a .^{51}$ reported that patients with a poststroke SBP of $150 \mathrm{~mm} \mathrm{Hg}$ or greater had a significantly higher stroke recurrence rate than those with poststroke SBP below $150 \mathrm{~mm}$ $\mathrm{Hg}$. Although not specifically assessed in our patients, isolated systolic hypertension (ISH) is common in the elderly, ${ }^{52}$ and 36-year follow-up data from the Framingham Study has shown stroke incidence to be significantly greater in elderly patients with ISH. ${ }^{53}$ The Systolic Hypertension in the Elderly Program (SHEP) has provided further support for a critical role of ISH in stroke, with its finding that stepped-care antihypertensive drug treatment of ISH in patients $>60$ years of age significantly reduced the five-year incidence of stroke. ${ }^{54}$ The stronger effect of SBP compared to DBP in predicting recurrent stroke in our sample parallels its disproportionate effect in the prediction of stroke ocurrence and stroke-related mortality. ${ }^{52,55,56}$

In case-control studies, heavy alcohol intake has been reported to be an independent risk factor for both hemorrhagic and ischemic stroke. ${ }^{26,27}$ Heavy alcohol use was reported to be an independent risk factor for stroke recurrence within 5 years of index stroke in the Northern Manhattan Stroke Study. ${ }^{3}$ Several epidemiologic studies have suggested a $J$-shaped relationship between alcohol consumption and initial ischemic stroke, with low to moderate levels of alcohol intake having a protective effect against the occurrence of stroke and heavy intake elevating the risk of a cerebrovascular event. ${ }^{26,27,57}$ We were unable to confirm a $\mathrm{J}$-shaped association between alcohol consumption and the risk of recurrent stroke in our sample. Alcohol intake exceeding 160 grams per week did result in an increased risk of stroke recurrence but low to moderate levels of alcohol consumption (60 to 160 grams per week) were not associated with a protective effect. While our data showed that 29 of 157 nondrinkers (18.5\%) and 5 of 35 light to moderate drinkers (14.3\%) experienced a recurrence over the follow-up period versus
10 of 48 heavy drinkers (20.8\%), suggesting a possible protective effect in the light to moderate drinking group, small sample sizes in the light to moderate and heavy drinking groups may have hampered our ability to recognize significant effects. Our reliance on self-reports of alcohol intake may also have resulted in an underestimation of the true effect of heavy alcohol use in relation to the risk of stroke recurrence.

Although they were treated primarily as covariates in our study, the effects of sex and education on stroke recurrence deserve further comment. We found sex to be a significant independent predictor of stroke recurrence, with women at elevated risk. Of the previous studies of factors influencing the risk of recurrent stroke, ${ }^{2-4,6,23,22,43,58}$ only the Framingham study ${ }^{1}$ reported an effect for gender, with men having almost double the rate of recurrence observed in women. Substantial differences in study design and patient type in previous studies of stroke recurrence make meaningful comparisons between studies difficult. In our sample, further subanalyses revealed sex differences in the distribution of age and alcohol intake, with women tending to be older and men tending to be heavier drinkers, suggesting that an unequal distribution of stroke risk factors between the sexes may account for the higher rate of recurrence that we observed in women. This interesting finding needs further study to determine whether identified vascular risk factors interact with gender to influence stroke recurrence. Although we did not have the necessary data in our sample, age at menopause and estrogen status ${ }^{59}$ are additional factors that may affect stroke recurrence in women and warrant investigation in future studies. Education was of borderline significance in our multivariate analyses, with less educated patients experiencing a higher rate of recurrence. The effect of education on stroke recurrence has not been previously examined and our finding may reflect poorer control of risk factors in the less educated patients. After a first stroke, patients with less education may not be aware of the substantial risk of recurrent stroke, with resultant failure to modify diet or risk behaviors such as smoking and alcohol use. Among the less educated, lower socioeconomic status with resultant reduced access to regular health care may also affect control of vascular risk factors and may be an explanation for their increased susceptibility to stroke recurrence.

The limitations of our study include an inability to investigate infarct volume, silent brain infarction, and leukoaraiosis as predictors of recurrence due to a lack of quantitative neuroimaging, as well as a restricted sample size which limited our ability to reliably investigate the association between the clinical subtype of dementia (e.g., $\mathrm{AD}$ with stroke, $\mathrm{VaD}$ ) and risk of stroke recurrence. Although we might infer that patients with $\mathrm{VaD}$ would be at greater risk of stroke recurrence than those with mixed dementia due to a greater burden of cerebrovascular disease, our results suggest that the dementia syndrome it- 
self would be likely to remain an important determinant of recurrent stroke, independent of presumed etiology, because dementia remained a significant independent predictor of stroke recurrence when we explored risk factors for cerebrovascular disease and characteristics of the index stroke in our multivariate models. We also lacked reliable information on compliance with prescribed therapies and medical follow-up in our sample. We plan to continue to follow our original cohort, and we are recruiting a second cohort to expand our observations regarding dementia and recurrent stroke, including the relationship between apolipoprotein $E$ genotype and risk of stroke recurrence. Further prospective studies of stroke patients are warranted to investigate the role of silent stroke, larger infarct volumes, and cerebral white matter disease as predictors of stroke recurrence and how they contribute to the pathogenesis of stroke-related cognitive decline.

We found that dementia following stroke is a potent risk factor for recurrent stroke, suggesting that careful attention should be paid to the detection of cognitive impairment in stroke patients. The recognition of dementia in stroke survivors may identify a subgroup at higher risk for recurrence, providing an opportunity for targeted intervention. For example, the institution of supervised treatment programs for stroke survivors with dementia may lower rates of stroke recurrence in this group. Our results also support prompt evaluation and treatment of cardiac disease in stroke survivors to prevent recurrent stroke due to cardiogenic cerebral embolism. Education of stroke patients and their families, in particular those who are less educated or from lower socioeconomic strata, may increase their awareness of risk factors for stroke recurrence, encourage the modification of risk-prone behaviors, and improve compliance with medical therapies, leading to a better outcome following ischemic stroke.

\section{Acknowledgments}

We thank the staff of the Stroke and Aging Research Project for their efforts, and Drs. Richard Mayeux, J.P. Mohr, and Ralph L. Sacco for their helpful comments on the manuscript.

\section{References}

1. Sacco RL, Wolf PA, Kannel WB, McNamara PN. Survival and recurrence following stroke. Stroke 1982;13:290-295.

2. Hier DB, Foulkes MA, Swiontoniowski M, et al. Stroke recurrence within 2 years after ischemic infarction. Stroke 1991;22: 155-161.

3. Sacco RL, Shi T, Zamanillo MC, Kargman DE. Predictors of mortality and recurrence after hospitalized cerebral infarction in an urban community: The Northern Manhattan Stroke Study. Neurology 1994;44:626-634.

4. Burn J, Dennis M, Bamford J, Sandercock P, Wade D, Warlow C. Long-term risk of recurrent stroke after a first-ever stroke. The Oxfordshire Community Stroke Project. Stroke 1994;25: 333-337.

5. Garraway WM, Whisnant JP. The changing pattern of hypertension and the declining incidence of stroke. JAMA 1987;258: 214.

6. Meissner I, Whisnant JP, Garraway WM. Hypertension management and stroke recurrence in a community (Rochester, Minnesota, 1950-1979). Stroke 1988;19:459-463.
7. Tatemichi TK, Desmond DW, Mayeux R, et al. Dementia after stroke: baseline frequency, risks, and clinical features in a hospitalized cohort. Neurology 1992;42:1185-1193.

8. Tatemichi TK, Paik M, Bagiella E, et al. Risk of dementia after stroke in a hospitalized cohort: results of a longitudinal study. Neurology 1994;44:1885-1891.

9. Kokmen E, Whisnant JP, O'Fallon WM, Chu CP, Beard CM. Dementia after ischemic stroke: a population-based study in Rochester, Minnesota (1960-1984). Neurology 1996;19:154159.

10. Tatemichi TK, Paik M, Bagiella E, Desmond DW, Pirro M, Hanzawa L. Dementia after stroke is a predictor of long-term survival. Stroke 1994;25:1915-1919.

11. Goodglass H, Kaplan E. The assessment of aphasia and related disorders, 2nd ed. Philadelphia: Lea \& Febiger, 1983.

12. Folstein MF, Folstein SE, McHugh PR. Mini-mental state: a practical method for grading the cognitive state of patients for the clinician. J Psychiatr Res 1975;2:189-198.

13. Mahoney FI, Barthel DW. Functional evaluation: Barthel index. Md Med J 1965;14:61-65.

14. Blessed G, Tomlinson BE, Roth $M$. The association between quantitative measures of dementia and of senile change in the cerebral grey matter of elderly subjects. Br J Psychiatry 1968; 114:797-811.

15. Stern Y, Andrews H, Pittman J, et al. Diagnosis of dementia in a heterogeneous population: Part 1. Development of a neuropsychological paradigm and quantified correction for education. Arch Neurol 1992;49:453-460.

16. Tatemichi TK, Desmond DW, Stern Y, Paik M, Sano M, Bagiella E. Cognitive impairment after stroke: frequency, patterns, and relationship to functional abilities. $J$ Neurol Neurosurg Psychiatry 1994;57:202-207.

17. Skilbeck CE, Wade DT, Hewer RL, Wood VA. Recovery after stroke. J Neurol Neurosurg Psychiatry 1983;46:5-8.

18. Wade DT, Wood VA, Newer RL. Recovery after stroke-the first three months. J Neurol Neurosurg Psychiatry 1985;48:713.

19. American Psychiatric Association. Diagnostic and statistical manual of mental disorders, 3rd ed, revised. Washington, DC: American Psychiatric Association, 1987.

20. Hughes CP, Berg L, Danziger WL, Cohen LA, Martin RL. A new clinical scale for the staging of dementia. Br J Psychiatry 1982;140:566-572.

21. Foulkes MA, Wolf PA, Price TR, Mohr JP, Hier DB. The Stroke Data Bank: design, methods, and baseline characteristics. Stroke 1988;19:547-554.

22. Kaplan EL, Meier P. Nonparametric estimation from incomplete observations. J Am Stat Assoc 1958;53:457-481.

23. Alter M, Sobel E, McCoy RL, et al. Stroke in the Lehigh Valley: risk factors for recurrent stroke. Neurology 1987;37 503-507.

24. Chodosh EH, Foulkes MA, Kase CS, et al. Silent stroke in the NINCDS Stroke Data Bank. Neurology 1988;38:1674-1679.

25. Davis PH, Clarke WR, Bendixen BH, Adams HP, Woolson RF, Culebras A, and the TOAST investigators. Silent cerebral infarction in patients enrolled in the TOAST study. Neurology 1996;46:942-948.

26. Gill JS, Zezulka AV, Shipley MJ, Gill SK, Beevers DG. Stroke and alcohol consumption. N Engl J Med 1986;315:1041-1046.

27. Gill JS, Shipley MJ, Tsementziz SA, et al. Alcohol consumption: a risk factor for hemorrhagic and nonhemorrhagic stroke. Am J Med 1991;90:489-497.

28. Cox DR. Regression models and lifetables. J R Stat Soc B $1972 ; 34: 187-220$.

29. Terent A. Survival after stroke and transient ischemic attacks during the 1970's and 1980's. Stroke 1989;20:1320-1326.

30. Viitanen M, Eriksson S, Asplund K, Wester PO, Winblad B. Determinants of long-term mortality after stroke. Acta Med Scand 1987;221:349-356.

31. Dennis M, Burn J, Sandercock P, Bamford J, Wade D, Warlow C. Long-term survival after first-ever stroke. The Oxfordshire Community Stroke Project. Stroke 1993;24:796-800.

32. Marquardsen J. The natural history of acute cerebrovascular disease: a retrospective study of 769 patients. Acta Neurol Scand 1969;45(suppl 38):S11-S155.

33. Dombovy ML, Basford JF, Whisnant JP, Bergstralh EJ. Disability and use of rehabilitation services following stroke in Rochester, Minnesota, 1975-1979. Stroke 1987;18:830-836. 
34. Woo J, Kay R, Yuen YK, Nicholls MG. Factors influencing long-term survival and disability among three-month stroke survivors. Neuroepidemiology 1992;11:143-150.

35. Bonita R, Ford MA, Stewart AW. Predicting survival after stroke: a three-year follow-up. Stroke 1988;19:669-673.

36. Loeb C, Gandolfo C, Bino G. Intellectual impairment and cerebral lesions in multiple cerebral infarcts: a clinicalcomputed tomography study. Stroke 1988;19:560-565.

37. Liu CK, Miller BL, Cummings JL, et al. A quantitative MRI study of vascular dementia. Neurology 1992;42:138-143.

38. Tatemichi TK, Foulkes MA, Mohr JP, et al. Dementia in stroke survivors in the Stroke Data Bank cohort. Prevalence, incidence, risk factors, and computed tomographic findings. Stroke 1990;21:858-866.

39. Tatemichi TK, Desmond DW, Paik M, et al. Clinical determinants of dementia related to stroke. Ann Neurol 1993;33: 568-575.

40. Ferrucci L, Guralnik JM, Salive ME, et al. Cognitive impairment and risk of stroke in the older population. J Am Geriatr Soc 1996;44:237-241.

41. Tatemichi TK, Kase CS, Bagiella E, Desmond DW, Mohr JP. Silent brain infarction and dementia in the Stroke Data Bank [abstract]. Ann Neurol 1994;36:265.

42. Figueroa M, Tatemichi TK, Desmond DW, Cross DT. CT cor relates of dementia in lacunar infarction [abstract]. Ann Neurol 1992;32:206

43. EAFT Study Group. Silent brain infarction in nonrheumatic atrial fibrillation. Neurology 1996;46:159-165.

44. Miyao S, Takano A, Teramoto J, Takahashi A. Leukoaraiosis in relation to prognosis for patients with lacunar infarction. Stroke 1992;23:1434-1438.

45. van Swieten JC, Kapelle IJ, Algra A, van Latum JC, Koudstaal PJ, van Gijn J, for the Dutch TIA trial study group. Hypodensity of the cerebral white matter in patients with transient ischemic attack or minor stroke: influence on the rate of subsequent stroke. Ann Neurol 1992;32:177-183.

46. Davis PH, Dambrosia JM, Schoenberg BS, et al. Risk factors for ischemic stroke: a prospective study in Rochester, Minnesota. Ann Neurol 1987;22:319-327.

47. Broderick JP, Phillips SJ, O'Fallon M, Frye RL, Whisnant JP. Relationship of cardiac disease to stroke occurrence, recurrence, and mortality. Stroke 1992;23:1250-1256.
48. Sacco RL, Hauser WA, Mohr JP, Foulkes MA. One-year outcome after cerebral infarction in whites, blacks, and hispanics. Stroke 1991;22:305-311.

49. DeRook FA, Beach KW, Lytle NJ, Golby AJ, Albers GW Transesophageal echocardiography and carotid ultrasound in patients with cerebral ischemia: prevalence of findings and recurrent stroke risk. J Am Coll Cardiol 1994;23:1598-1603.

50. Bogousslavsky J, Castillo V, Regli F, Xavier J, Sylvain G. Stroke recurrence in patients with patent foramen ovale: a prospective study of 140 patients [abstract]. Stroke 1996;27: 166.

51. Irie $\mathrm{K}$, Yamaguchi $\mathrm{T}$, Minematsu $\mathrm{K}$, Omae $\mathrm{T}$. The J-curve phenomenon in stroke recurrence. Stroke 1993;24:1844-1849.

52. Kannel WB, Wolf PA, Castelli WP, et al. Systolic blood pressure, arterial rigidity, and risk of stroke: the Framingham Study. JAMA 1981;245:1225.

53. Wolf PA, Cobb JL, D'Agostino RB. Epidemiology of stroke. In Barnett HJM, Mohr JP, Stein BM, et al, eds. Stroke: pathophysiology, diagnosis, and management. 2nd ed. New York: Churchill Livingstone, 1992:3-27.

54. The SHEP Cooperative Research Group. Prevention of stroke by antihypertensive drug treatment in older persons with isolated systolic hypertension: final results of the Systolic Hypertension in the Elderly Program (SHEP). JAMA 1991;265: 3255-3264.

55. Rabkin SW, Mathewson FAL, Tate RB. The relation of blood pressure to stroke prognosis. Ann Int Med 1978;89:15-20.

56. Terent A, Andersson B. The prognosis for patients with cerebrovascular stroke and transient ischemic attacks. Ups J Med Sci 1981;86:63-74.

57. Palomalki $H$, Kaste M. Regular light-to-moderate intake of alcohol and the risk of ischemic stroke: Is there a beneficial effect? Stroke 1993;24:1828-1832.

58. Viitanen M, Eriksson S, Asplund K. Risk of recurrent stroke, myocardial infarction and epilepsy during long-term follow-up after stroke. Eur Neurol 1988;28:227-231.

59. Furberg CD, Shemanski L, Psaty BM, O'Leary DH, Tracy RP, Bush TL. Associations of postmenopausal estrogen use with cardiovascular disease and its risk factors in older women. The CHS Collaborative Research Group. Circulation 1993;88: 2163-2171. 